\title{
Fusion framework for Robust and Secured Watermarking
}

\author{
Nisha Sharma \\ IEEE Student Member, PhD \\ Scholar, Punjab Technical \\ University, Punjab, India
}

\author{
Anjali Goyal \\ Asst. Professor, Department of \\ Computer Applications, GNIMT, \\ Punjab, India
}

\author{
Y.S Brar \\ Professor, Department of \\ Electrical Engineering, GNDEC, \\ Punjab, India
}

\begin{abstract}
This paper presents a robust and secure watermarking technique for digital image. To implement the technique, Discrete Wavelet Transform (DWT) is applied on cover image. Further on Low-Low (LL) sub-band of DWT, Discrete Cosine Transform (DCT) is applied which is followed by Singular Value Decomposition (SVD). To introduce the secure watermarking, watermark is secured using Arnold Transformation and embedded in the cover image. Parameters such as Peak Signal to Noise Ratio (PSNR) and Normalized Correlation (NC) are used for checking the reliability of the proposed technique. Different attacks like noise, filtering, rotation, cropping, flipping, and compression are applied on watermarked image to check the robustness of the proposed approach.
\end{abstract}

\section{Keywords}

Watermarking, DWT, DCT, SVD, Arnold Transformation

\section{INTRODUCTION}

Digital data today is a word that everyone is aware of; as the use of digital data has become a part of every individual's life. Letters today are replaced by emails or instant messages. Hard copied photographs are rarely used now. Instead digital images are in use as they are easy to transmit from one place to other around the world. This ease of handling digital media has made it prone to many issues such as hacking, illegal copying, pirating, tampering etc. Watermarking of digital media can be used to set up the originality of such images, audios and videos. Numerous techniques have been proposed till date but each approach owns some advantages and disadvantages from the point of security, capacity and robustness. Watermarking can be defined as a process in which some ownership or special data i.e. text/image/signal is embedded in a multimedia content in such a manner so that original data is protected from various attacks [1].

Watermarking techniques can be classified into spatial domain and frequency domain. Spatial domain watermarking techniques are based on direct embedding of watermark by slightly modifying the pixels or subsets of cover image. Many methods related to spatial domain have been given such as Least Significant Bit (LSB) insertion [2], Patchwork scheme [3], Correlation based technique [4-6], Pre-Filtering technique[7] etc.

Frequency or Transform domain watermarking techniques are more robust as compared to spatial domain watermarking techniques as the watermark is embedded in the frequency bands rather than directly to the pixels. Frequency domain techniques are preferred because of their robustness towards cropping, contrast enhancement, blurring and low pass filtering attacks. First global Discrete Cosine Transformation(DCT) watermarking was proposed by Cox et al. [8] which was basically designed to bear compression attacks. Tao and Dickinson [9] embedded watermark in luminance domain by selecting blocks of DCT. Hsu and Wu [10] inserted Gaussian vector in the mid frequency band of DCT to bear cropping, enhancement and compression attacks, Huang et al. [11] inserted watermark in Direct Current (DC) components by using luminance texture masking. Wong et al. [12] also proposed a similar technique but band-pass filtering was used in place of luminance texture masking. Huang and Guan[13] used DCT and Singular Value Decomposition (SVD) based watermarking strategy for achieving highest robustness without losing transparency. Zhao et al. [14] applied the concept of threshold for watermarking and presented a technique with good imperceptibility and robustness. Naik and Holambe [15] presented blind watermarking technique based on adding entire watermark image by changing DCT coefficients of cover image to add odd or even determined by the DCT coefficients of watermark image. This technique has basically provided biometric image compression and authentication. Foo and Dong [16] proposed a blind and efficient watermarking technique based on block DCT and SVD by adjustments on watermark strength using adaptive frequency mask. Their approach was robust to various image processing operations and geometric attacks. Kundur and Hazinakos [17] presented image fusion Discrete Wavelet Transformation (DWT) watermarking technique based on salient features measures by adding of watermark bits repeatedly in the DCT coefficients of host image depending upon the selection done by the randomly selected key and then extended their research work in [18] by using Fusemark watermarking in multi resolution data fusion principles considering the Human Visual System (HVS) properties of an image. Correlation coefficient was used to access watermark robustness. Lu et al. [19] brought the concept of 'cocktail watermarking' where dual complimentary watermarks were added in DWT domain and regardless of attack, one watermark could be detected. Inspired by these authors, Raval and Rege [20] also described that watermark added in low frequency component is robust against low pass filtering, geometric distortions and compression whereas, watermark added in high frequency components is robust against histogram equalization and cropping attacks. Ganic and Eskicioglu [21] enhanced the technique proposed by Raval and Rege by adding watermark to SVD domain of low and high frequency components to remove the visibility limitation. Song and Zhang [22] proposed DWT and SVD based watermarking technique using Tent chaotic mapping for encryption of watermark. Their technique proved better in terms of quality watermarked image and robust to wide range of attacks.

Laskar et al. [23] proposed a DCT and DWT based watermarking technique with good imperceptibility and higher robustness. Divecha and Jani [24] proposed a DCT-DWT and SVD based watermarking technique satisfying the trade off 
between imperceptibility and robustness along with very high data hiding capacity. Khan et al. [25] proposed a DWT-DCTSVD based watermarking technique using zigzag mapping of DCT coefficients in the High-High $(\mathrm{HH})$ band of DWT. Saxena et al. [26] proposed embedding of watermark in DWTDCT-SVD using trigonometric function and obtained high PSNR values with high robustness to various image processing attacks. Singh et al[27] presented a hybrid scheme of DWTDCT transformation of images and then inserting singular values of watermark into singular values of host image and is quite robust to many attacks. Naik and Pal[28] introduced a partial image cryptosystem using DCT and Arnold Transformation in which DCT is applied to each colour band of colour image and then the coefficients are selected and encrypted with Arnold Transformation and then are embedded with the help of some secret key and the results describes it to be very secure.

The present paper makes use of DWT, DCT and SVD to present fusion framework for robust watermarking. Section 2 presents various descriptors and parameters used in the proposed framework. Section 3 describes the proposed algorithm. Section 4 highlights results based on experimental investigations. Conclusions are presented in Section 5.

\section{DESCRIPTORS USED}

\subsection{Discrete Wavelet Transformation (DWT)}

DWT is a local property technique that uses distinct high and low frequencies to analyse the image using wavelet and scaling functions. DWT separates an image into approximations and details of an image which are described as LL (Approximation Coefficients), HL (Horizontal Details), LH (Vertical Details) and HH (Diagonal Details).

LL band contains the image much closer to the original image and maximum energy is concentrated here. Whereas all the other 3 bands contain the edge detail, upright detail and texture detail which may be good for increasing capacity of watermarking bits but on the other hand it may inhibit robustness. Scaling is used to further refine the image. The technique can be visualized as shown in Figure 1:

\begin{tabular}{|c|c|}
\hline LL & HL \\
\hline LH & HH \\
\hline
\end{tabular}

(a)

\begin{tabular}{|c|c|c|}
\hline $\mathrm{LL}_{1}$ & $\mathrm{HL}_{1}$ & \multirow{2}{*}{$\mathrm{HL}_{2}$} \\
\cline { 1 - 2 } $\mathrm{LH}_{1}$ & $\mathrm{HH}_{1}$ & \\
\hline & & \\
$\mathrm{LH}_{2}$ & $\mathrm{HH}_{2}$ \\
& & \\
\hline \multicolumn{2}{|c|}{ (b) } \\
\hline
\end{tabular}

Fig 1: (a) 1- level 2D-DWT (b) 2-level 2D-DWT

\subsection{Discrete Cosine Transformation (DCT)}

DCT is a digital signal process technique in which an image is linearly transformed into frequency domain such that the maximum energy is clustered into few low frequency components of DCT based upon the data correlation. This concentration of energy not only centralizes the information but also minimizes the restricting effect thus making it superior for compression. The elements stored at location $(1,1)$ are the Direct current (DC) components whereas rest of them are Alternate Current (AC) components. The gray area in Fig. 2 shows the middle range frequency elements of DCT matrix.
Image or sub image is first converted into its DCT equivalent then the required modifications are done. After all the amendments, inverse DCT is applied on image. 2D DCT is computed using Eq. (1):

$$
\begin{aligned}
& F(i j)= \\
& a(i) a(j) \sum_{m=0}^{N-1} \sum_{n=0}^{N-1} f(m n) \cos \left[\frac{2(m+1) i \Pi}{2 N}\right] \cos \left[\frac{2(m+1) j \Pi}{2 N}\right]
\end{aligned}
$$

whereas 2D inverse DCT is computed through Eq. (2):

$$
\begin{aligned}
& f(m n)= \\
& \sum_{m=0}^{N-1} \sum_{n=0}^{N-1} a(i) a(j) F(i j) \cos \left[\frac{2(m+1) i \Pi}{2 N}\right] \cos \left[\frac{2(m+1) j \Pi}{2 N}\right]
\end{aligned}
$$

The image frequencies for $8 \times 8$ block obtained through $2 \mathrm{D}$ DCT as described in Figure 2.

Low to High

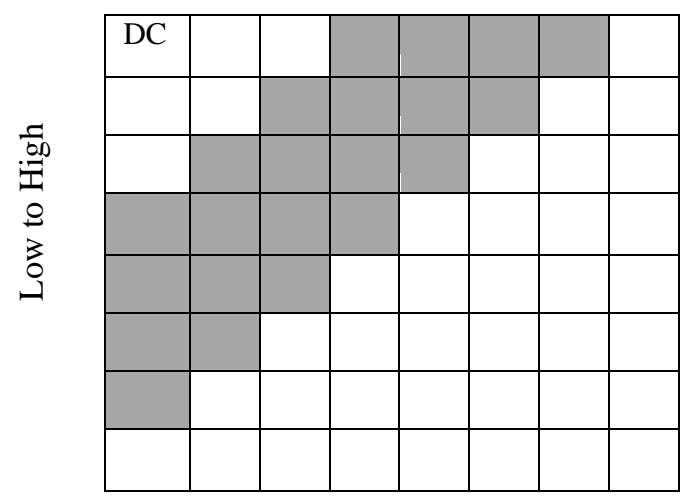

Fig 2: Energy spread of DC and AC components

The spread of energy is in such a manner that upper left corner contains the low signal energy of the image and proceeding downwards to the lower right corner the high signal energy is obtained. Embedding watermark in the higher and lower signal energies always possess a conflicting behaviour for robustness of watermark i.e. embedding watermark in low frequency components have greater robustness to low filtering, compression and geometric attacks where as that in high frequency components are robust against cropping and histogram equalization attacks hence considering the trade-off, usually middle frequency band is selected for embedding the watermark.

\subsection{Singular Value Decomposition (SVD)}

SVD is a factorizing tool for real and complex matrices and is used in many fields of digital image and signal processing. SVD transformation decomposes an image $I_{m x n}$ into two orthogonal matrices $U_{m x m}$ and $V_{n x n}$ and a diagonal matrix $S_{m x n}$ which contains the singular values, thus it is called singular matrix and specifies the luminance value of image. Whereas $U$ and $V$ matrices present the geometry of the image thus eventually are called non singular matrices. This decomposition can be represented by Eq. (3):

$$
U S V=S v d(I)
$$

Whereas Inverse SVD can be represented by Eq. (4):

$$
I_{m \times n}=U_{m \times m} S_{m \times n} V_{n \times n}^{T}
$$


SVD is widely used in watermarking because of its intrinsic algebraic properties and good stability which can be judged by the fact that addition of some amount of external data don't change the singular values. Moreover these values are the least affected by attacks such as compression, noise etc. which makes it an efficient tool to increase the robustness of watermark. The $S_{m \times n}$ can be represented mathematically through Eq. (5):

$$
S_{m \times n}=\begin{array}{ccc}
S_{1} & 0 & 0 \\
0 & \ddots & 0 \\
0 & 0 & S_{m n}
\end{array}
$$

\subsection{Arnold Transformation (AT)}

It is an encrypting tool which is used in image watermarking to scramble the watermark so that even if it is extracted by unauthorized users, they could not be able to recognize it as it needs an inverse Arnold transformation function to decode the extracted watermark. It was given by Vladimir Arnold in 1960s [29]. It basically ruptures the correlation of data resulting to decoded image that does not hinder the transmission and extraction of watermark hence ensures a secure and robust detection of watermark.

Arnold Transformation (AT) for an $N \times N$ image can be defined using Eq. (6):

$$
\left(\begin{array}{l}
x^{\prime} \\
y^{\prime}
\end{array}\right)=\left(\begin{array}{ll}
1 & 1 \\
1 & 2
\end{array}\right)\left(\begin{array}{l}
x \\
y
\end{array}\right)(\bmod N)
$$

Here $x^{\prime}$ and $y^{\prime}$ are scrambled pixels of original $(x, y)$ pixels The Arnold transformation is performed iteratively to obtain the decoded image. This iterative term is called Arnold periodicity. The Arnold periodicity used in the proposed algorithm in this paper is 5 . The image has to be inverse Arnold transformed with the same number of iterations that were used during encoding so that a decoded image can be obtained.

\subsection{Peak Signal to Noise Ratio (PSNR)}

PSNR is defined as power of a signal to corrupted signal. It is most commonly used as a quality measure for reconstructed images in image processing. An image when undergoes any kind of modifications and then are reconstructed, PSNR expressed in Decibels $(\mathrm{Db})$ gives the quality of the image. Usually a PSNR value in the range of $30-60 \mathrm{Db}$ is considered to be ideal. It is assumed that more is the value of PSNR, better is the quality.

Mathematically PSNR is given by Eq. (7):

$$
P S N R=10 \log _{10} \frac{255^{2}}{M S E}
$$

Where MSE is mean square error i.e. average of squares of errors.

\subsection{Bit Error Rate (BER)}

BER is defined as the ratio of number of bit errors to the total number of transmitted bits given by Eq. (8). As per watermarking theory, it is described as total number of incorrectly detected watermark bits to total number of embedded bits. The more, it is closer to zero, the more accurate are the results.
We have stated the value of PSNR to study the reliability of the proposed technique which can also used to calculate BER as it is inversely proportional to PSNR and can be represented as Eq. (9):

$$
B E R=\frac{1}{P S N R}
$$

\subsection{Normalised Correlation Coefficient (NC)}

$\mathrm{NC}$ is also a quality measure which is used in image processing to present the correlation between original and modified or attacked image. $\mathrm{NC}$ is used to measure correctness of extracted watermark. It is defined by Eq. (10):

$$
N C=\frac{1}{W_{h} \times W_{w}} \sum_{i=0}^{W_{h}-1} \sum_{j=0}^{W_{w}-1} W(i, j) \times W^{\prime}(i, j)
$$

$W_{h}$ and $W_{w}$ are the height and width of watermark respectively. $W(i, j)$ and $W^{\prime}(i, j)$ are the pixels at $(i, j)$ location. The value of NC lies between -1 and 1 . The more is the Positive correlation, better are the results.

\section{PROPOSED TECHNIQUE}

The Proposed technique implements a secured watermark embedded using DWT-DCT-SVD. The process of embedding a watermark and extraction of watermark is shown algorithmically and graphically in this section.

\subsection{Watermark Embedding}

The steps used by the proposed technique are as:

1. Resize the cover gray scale image I to $512 \times 512$ pixels image and perform 1-level DWT and select the LL band.

2. Divide the LL band into blocks of $8 \times 8$ and perform $2 \mathrm{D}$ DCT on each block.

3. Now select $(\mathrm{k}, \mathrm{k})$ bits of each block and create a matrix and apply SVD on I to obtain $\mathrm{U}_{1}, \mathrm{~S}_{1}$ and $\mathrm{V}_{1}$.

4. Read the $32 \times 32$ watermark image $\mathrm{W}$ and perform Arnold Transform (AT) upon W to encode it.

5. Embed the watermark bits into the singular values of I with some scaling factor $f$ using Eq. (11)

$$
\mathrm{S}_{1}{ }^{\prime}=\mathrm{S}_{1}+\mathrm{f} \times \mathrm{W}
$$

6. Perform SVD again on the embedded bits and obtain the $\mathrm{U}_{2}, \mathrm{~S}_{2}$ and $\mathrm{V}_{2}$.

7. Perform inverse SVD on $S_{1}$ ' with orthogonal matrices of cover image i.e. $\mathrm{S}=\mathrm{U}_{1} \mathrm{~S}_{1}{ }^{\prime} \mathrm{V}_{1}{ }^{\mathrm{T}}$

8. Rewrite the $(\mathrm{k}, \mathrm{k})$ values of each block by replacing it by $\mathrm{S}$.

9. Perform inverse DCT and inverse DWT to get the resultant Watermarked Image WI.

\subsection{Diagrammatical Representation of Watermark Embedding}

$$
B E R=\frac{\text { Number of incorrect bits }}{\text { Total number of transmitted bits }}
$$




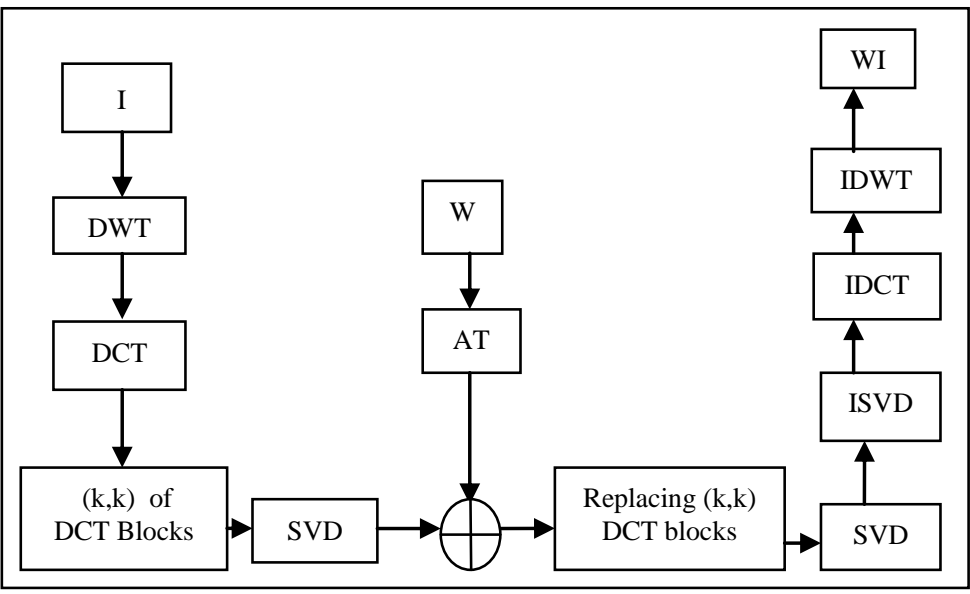

Fig 3: Block Diagram to embed watermark

\subsection{Watermark Extraction}

The steps to be followed in watermark extraction are as:

1. Read the watermarked image WI and resize it to $512 \times 512$ pixels if needed.

2. Perform 2D DWT and select the LL band.

3. Divide the image into $8 \times 8$ blocks and apply 2D DCT on each block.

4. Select the $(k, k)$ elements of each block and create a matrix.

5. Apply SVD on the matrix obtained in step 4 to achieve orthogonal matrices $\mathrm{U}_{3}$ and $\mathrm{V}_{3}$ and singular value matrix $\mathrm{S}_{3 \text {. }}$.

6. Now apply Inverse SVD on Singular values extracted in step (5) with orthogonal matrices $\mathrm{U}_{2}$ and $\mathrm{V}_{2}{ }^{\prime}\left(\mathrm{U}_{2}\right.$ and $\mathrm{V}_{2}$ are the orthogonal matrices of watermarked image)

$$
\mathrm{WI}^{\prime}=\mathrm{U}_{2} \mathrm{~S}_{3} \mathrm{~V}_{2}^{\prime}
$$

7. Now extract the watermark bits by using Eq. (12)

$$
\mathrm{W}^{\prime}=\left(\mathrm{WI}^{\prime}-\mathrm{S}_{1}\right) / \mathrm{f}
$$

Here, $S_{1}$ is the singular value matrix of cover image.

8. Apply inverse Arnold Transform.

\subsection{Diagrammatical Representation of Watermark Extraction}

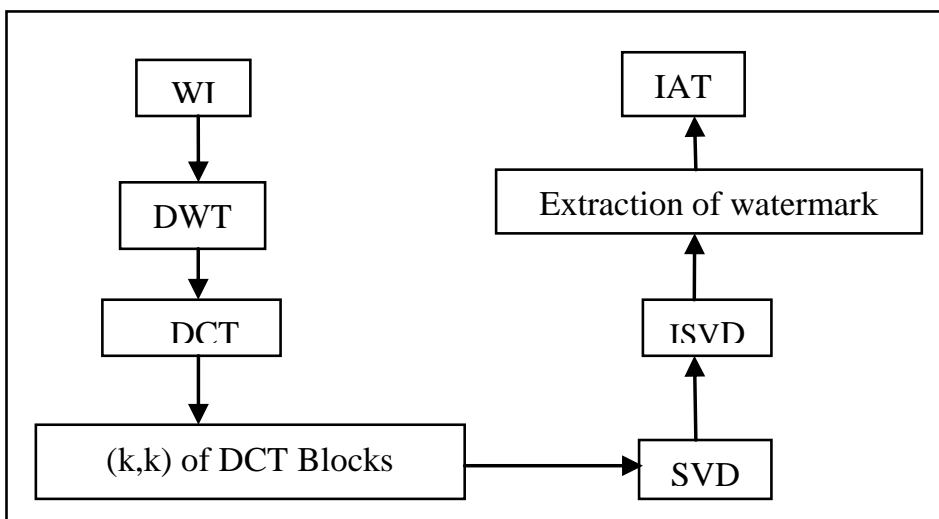

Fig 4: Block Diagram of Watermark Extraction.

\section{EXPERIMENTAL SETUP AND RESULTS}

The proposed technique involves study of watermark embedding and extraction at diagonal elements of cover image. As earlier stated, mid frequency bands of DCT are considered to hold a perfect balancing behaviour between robustness and transparency. As literature review depicts that most of the DCT based techniques use zigzag ordering of the elements followed by selection of some of elements using a secret key or some other phenomenon. The diagonal elements of mid and high frequency DCT coefficients are directly selected.

The results of watermark embedding at the diagonal elements of DCT block which are $(3,3)$ and $(4,4)$ lying in the mid frequency bands and $(5,5)$ and $(6,6)$ lying in the high frequency band are compared.

Figure 5(a) shows the cover image used for the experiments i.e. the standard image of Baboon in gray scale. Figure 5(b) shows the gray scale watermark image $(32 \times 32)$ and Figure $5(\mathrm{c})$ is the watermark image obtained after Arnold transformation at periodicity 5 .
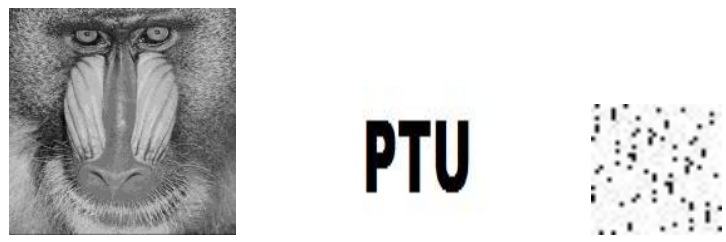

Figure 5: (a) Cover Image (b) Watermark Image (c) Arnold Transformed Watermark Image at periodicity 5.

The results using the proposed approach for Watermarked Image at different locations mentioned above with PSNR are shown in Table 1. The bold face value depicts that a particular location returns the best results.

Table 1. PSNR and Normalized Correlation values of

\begin{tabular}{|c|c|c|c|c|}
\hline $\begin{array}{l}\text { Locati } \\
\text { on }\end{array}$ & $\begin{array}{c}\text { Watermarked } \\
\text { image }\end{array}$ & PSNR & $\begin{array}{l}\text { Extracted } \\
\text { watermark }\end{array}$ & NC \\
\hline$(3,3)$ & & 49.3240 & & 0.9916 \\
\hline$(4,4)$ & & 49.1169 & & 0.9968 \\
\hline$(5,5)$ & & 48.9705 & & 0.9896 \\
\hline
\end{tabular}
extracted Watermark w.r.t. different locations 


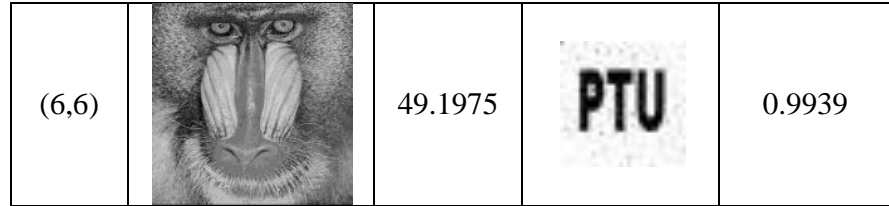

The results of watermarked image with different attacks are depicted in Table 2. Since attacks degrade the quality of the image, the only focus is on extraction of watermark even after such degradation of image. Hence, the NC values describe how close the extracted watermark is to the original watermark. While analysing Table 2 , it is inferred that middle frequency band are more robust as compared to high frequency bands. However diagonal elements taken from high frequency band are found to be outperforming for cropping and editing attacks.

Chart 1 depicts the PSNR values of watermarked image at different locations of watermark embedding. Similarly, Chart 2 shows the NC values of the extracted watermark with the original watermark image.

Chart 1. PSNR values at different locations.

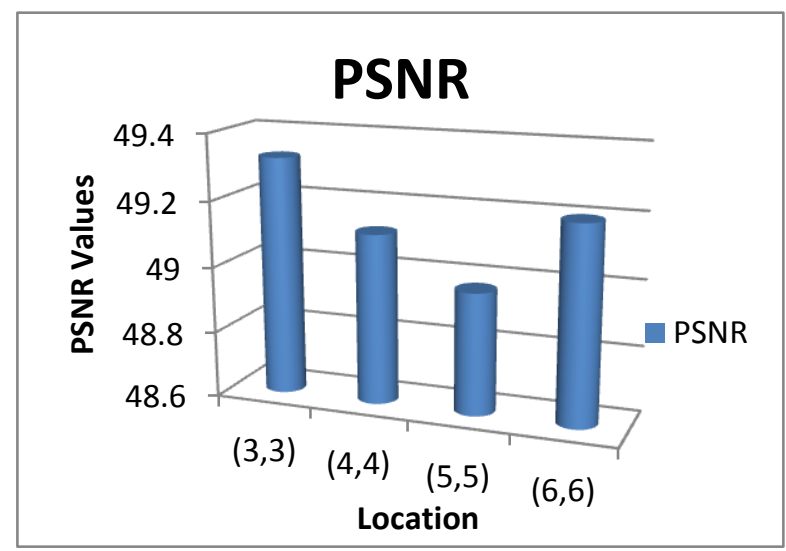

Chart 2. NC values at different locations.

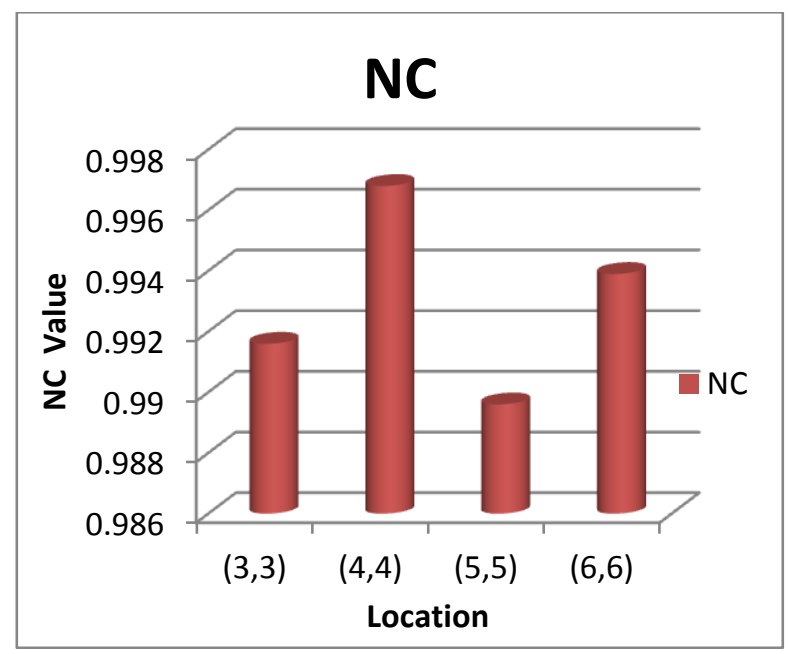

\section{CONCLUSION AND FUTURE WORK}

The paper presents a robust and secure watermarking technique using various transform domain oriented descriptors. The watermark is embedded after being secured using Arnold Transformation into cover image by the proposed approach. Watermark is embedded at different locations pertaining to mid frequency and high frequency bands. From the experimental work carried out in this paper, it is concluded that the proposed watermarking approach is quite robust as it is able to detect watermark even after various geometric, filtering, cropping and compression attacks. Through experiments, it is also observed that if the watermark is added to a certain position and the watermark is extracted from some other position it can be detected after inverse Arnold transformation but with lower normalized correlation. Arnold Transformation upon watermark ensures the security of watermark thus preventing it from getting accidently recovered. In future, this technique can be applied on colour images and on other image formats also.

\section{REFERENCES}

[1] Van Schyndel, R.G., Tirkel A.Z., and Osborne C.F., 1994. "A digital watermark." In Proceedings of IEEE International Conference in Image Processing, (ICIP-94) Vol. 2.

[2] Benoit, M.M, and Quisquater J.J., 1995 "Cryptology for digital TV broadcasting." Proceedings of the IEEE 83, No.6

[3] Bender W, Gruhl D, Morimoto N, Lu A , 1996 , "Techniques for data hiding." IBM systems journal 35, No. 3.4, pp: 313-336.

[4] Fridrich J, "Robust bit extraction from images." IEEE International Conference on Multimedia Computing and Systems, 1999. Vol. 2. IEEE, 1999.

[5] Pitas I and Kaskalis T. H., 1995, "Applying signatures on digital images."Proceedings of IEEE International Conference on Nonlinear Signal and Image Processing. Pp 460-463.

[6] Wolfgang RB., and Delp EJ. 1996, "A watermark for digital images." Proceedings of International Conference on Image Processing, 1996. Vol. 3.

[7] Johnson NF. and Katzenbeisser S, 2000 "A survey of steganographic techniques." Information hiding. Norwood, MA: Artech House.

[8] Cox I.J, Kilian J., Leighton F.T., and T. Shamoon T.,1997, "Secure spread spectrum watermnarking for multimedia" in IEEE Transactions on Image Processing, vol. 6 , no. 12 , pp:1673 -1687

[9] Tao B., Dickinson B.,1997, "Adaptive Watermarking in DCT domain", in Proceedings of IEEE International Conference on Acoustics, Speech and signal Processing,( ICASSP '97), vol.4, pp. 1985-2988

[10] Hsu CT, and Wu JL.,1999 "Hidden digital watermarks in images." , IEEE Transactions on Image Processing, Vol $8.1,: \mathrm{pp}: 58-68$

[11] Huang J, Shi YQ, and Shi Y, 2000,. "Embedding image watermarks in DC components.", IEEE Transactions on Circuits and Systems for Video Technology, vol. 10.6, pp: 974-979.

[12] Wong, P. H., Au, O. C., \& Wong, J. W. 2000. "Data hiding and watermarking in JPEG-compressed domain by DC coefficient modification". In Proceedings of SPIE, Security and Watermarking of Multimedia Contents II, Vol 3971.

[13] Huang F, and Guan ZH.,2004 "A hybrid SVD-DCT watermarking method based on LPSNR." Pattern 
Recognition Letters by Elsevier, Vol 25, No.15, pp: 1769-1775

[14] Zhao RM, Lian H, Pang HW and Hu B 2008, "A blind watermarking algorithm based on DCT." IEEE Second International Symposium on Intelligent Information Technology Application, (IITA'08).20-22, Vol. 3.

[15] Naik AK., and Holambe RS., 2010, "A blind DCT domain digital watermarking for biometric authentication." International Journal of Computer Applications, vol .1.16 pp: 11-15.

[16] Foo SW., and Dong Qi. ,2010, "A normalization-based robust image watermarking scheme using SVD and DCT." World Academy of Science, Engineering and Technology Vol:4 Pg:205-210.

[17] Kundur, D, and Hatzinakos D, 1998, "Digital watermarking using multiresolution wavelet decomposition." In Proceedings of IEEE International Conference on Acoustics, Speech and Signal Processing, Vol. 5.

[18] Kundur D, and Hatzinakos D. 2004, "Toward robust logo watermarking using multi resolution image fusion principles." IEEE Transactions on Multimedia, Vol. 6.1. pp: 185-198.

[19] Lu CS., Liao HYM, Huang SK , 2000, "Cocktail watermarking on images." Information Hiding. Springer Berlin Heidelberg.

[20] Raval, M. S., and Rege P. P., 2003, "Discrete wavelet transform based multiple watermarking scheme." TENCON 2003. In proceedings of IEEE Conference on Convergent Technologies for the AsiaPacific Region. Vol. 3.

[21] Ganic E, and Eskicioglu AM. 2004 "Robust DWT-SVD domain image watermarking: embedding data in all frequencies." Proceedings of the 2004 Workshop on Multimedia and Security. ACM.

[22] Song J, and Zhang Z., 2011, "A digital watermark method based on SVD in wavelet domain." International Journal of Advancements in Computing Technology (IJACT) Vol 3.8 pp: 205-214.

[23] Laskar, R. H., Choudhury M, Chakraborty K, 2011, "A Joint DWT-DCT Based Robust Digital Watermarking Algorithm for Ownership Verification of Digital Images." Computer Networks and Intelligent Computing. Springer Berlin Heidelberg, pp: 482-491.

[24] Divecha NH., and Jani NN., 2012, "Image Watermarking Algorithm using Dct, Dwt and Svd."In Proceedings of National Conference on Inovative Paradigm in Engineering and Technology (NCIPET-2012), International Journal of Computer Application Vol.13CA.

[25] Khan, M.I., Rahman, M., Sarker, M., \& Hasan, I., 2013. "Digital Watermarking for Image Authentication Based on Combined DCT, DWT and SVD Transformation". International Journal of Computer Science Issues, Vol. 10, Issue 3, No 1.

[26] Saxena, H, Saxena Praful, and Rastogi Shubham, 2014, "DWT-DCT-SVD based semi-blind reference image watermarking scheme using trignometric function."
International Journal of Conceptions on Computing and Information Technology Vol.2, Issue 2.

[27] Singh, A. K., Dave, M., \& Mohan, A. ,2014. Hybrid Technique for Robust and Imperceptible Image Watermarking in DWT-DCT-SVD Domain. Published in Springer, National Academy Science Letters 37, No. 4, pp 351-358.

[28] Naik, K., \& Pal, A. K. 2014. A Partial Image Cryptosystem Based on Discrete Cosine Transform and Arnold Transform. In Recent Advances in Information Technology pp. 65-73. Springer India.

[29] Zhang C, Wang J, and Wang W., 2008, "Digital image watermarking algorithm with double encryption by Arnold transform and logistic." In Proceedings of Fourth IEEE International Conference on. Networked Computing and Advanced Information Management (NCM'08). Vol. 1.

[30] Potdar, VM., Han S, and Chang E. 2005, "A survey of digital image watermarking techniques." In Proceedings of 3rd IEEE International Conference on Industrial Informatic,. (INDIN'05).

[31] Wang, B., Ding, J., Wen, Q., Liao, X., \& Liu, C. , 2009. "An image watermarking algorithm based on DWT DCT and SVD." In Proceedings of IEEE International Conference on Network Infrastructure and Digital Content, 2009. IC-NIDC 2009. pp. 1034-1038).IEEE. 
Table 2. Normalised Correlation of extracted watermarks from different locations after various attacks.

\begin{tabular}{|c|c|c|c|c|c|c|c|c|c|}
\hline \multirow{2}{*}{ Attack } & \multirow{2}{*}{$\begin{array}{c}\text { Attacked } \\
\text { Watermark Image }\end{array}$} & \multicolumn{2}{|c|}{ Attack Results at $(3,3)$} & \multicolumn{2}{|c|}{ Attack Results at $(\mathbf{4 , 4 )}$} & \multicolumn{2}{|c|}{ Attack Results at $(5,5)$} & \multicolumn{2}{|c|}{ Attack Results at $(6,6)$} \\
\hline & & $\begin{array}{l}\text { Extracted } \\
\text { watermark }\end{array}$ & NC & $\begin{array}{l}\text { Extracted } \\
\text { watermark }\end{array}$ & NC & $\begin{array}{l}\text { Extracted } \\
\text { watermark }\end{array}$ & NC & $\begin{array}{l}\text { Extracted } \\
\text { watermark }\end{array}$ & $\mathrm{NC}$ \\
\hline $\begin{array}{c}\text { Gaussian } \\
\text { noise } \\
(\mathrm{m}=0.01 \& \\
\mathrm{v}=0.01)\end{array}$ & & & 0.9318 & & 0.9555 & & 0.9516 & & 0.9416 \\
\hline $\begin{array}{c}\text { Speckle Noise } \\
(0.06)\end{array}$ & & & 0.9448 & & 0.9094 & & 0.9349 & & 0.9164 \\
\hline $\begin{array}{c}\text { Salt \& Pepper } \\
\text { noise } 0.05\end{array}$ & & & 0.9229 & & 0.9014 & & 0.9207 & & 0.9510 \\
\hline $\begin{array}{c}\text { Average filter } \\
{[3,3]}\end{array}$ & & & 0.8951 & & 0.8850 & & 0.8758 & & 0.8784 \\
\hline $\begin{array}{l}\text { Gaussian } \\
\text { filter[3,3] \% }\end{array}$ & & & 0.9190 & & 0.9153 & & 0.9017 & & 0.9055 \\
\hline Prewitt filter & & & 0.9317 & & 0.9265 & & 0.9155 & & 0.9534 \\
\hline Sobel filter & & & 0.8958 & & 0.9104 & & 0.9032 & & 0.9425 \\
\hline
\end{tabular}

Table 2. Normalised Correlation of extracted watermarks from different locations after various attacks. (continued)

\begin{tabular}{|c|c|c|c|c|c|c|c|c|c|}
\hline \multirow{2}{*}{ Attack } & \multirow{2}{*}{$\begin{array}{c}\text { Attacked } \\
\text { Watermark Image }\end{array}$} & \multicolumn{2}{|c|}{ Attack Results at $(3,3)$} & \multicolumn{2}{|c|}{ Attack Results at $(4,4)$} & \multicolumn{2}{|c|}{ Attack Results at $(5,5)$} & \multicolumn{2}{|c|}{ Attack Results at $(6,6)$} \\
\hline & & $\begin{array}{l}\text { Extracted } \\
\text { watermark }\end{array}$ & $\mathrm{NC}$ & $\begin{array}{l}\text { Extracted } \\
\text { watermark }\end{array}$ & NC & $\begin{array}{l}\text { Extracted } \\
\text { watermark }\end{array}$ & $\mathrm{NC}$ & $\begin{array}{l}\text { Extracted } \\
\text { watermark }\end{array}$ & $\mathrm{NC}$ \\
\hline $\begin{array}{c}\text { Rotation } 10 \\
\text { degree }\end{array}$ & & 3 & 0.8483 & & 0.8965 & ify & 0.8474 & Pif & 0.8560 \\
\hline $\begin{array}{c}\text { Rotation } 45 \\
\text { degree }\end{array}$ & & & 0.9502 & 3 & 0.8612 & 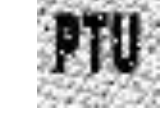 & 0.8557 & sidit & 0.8235 \\
\hline
\end{tabular}




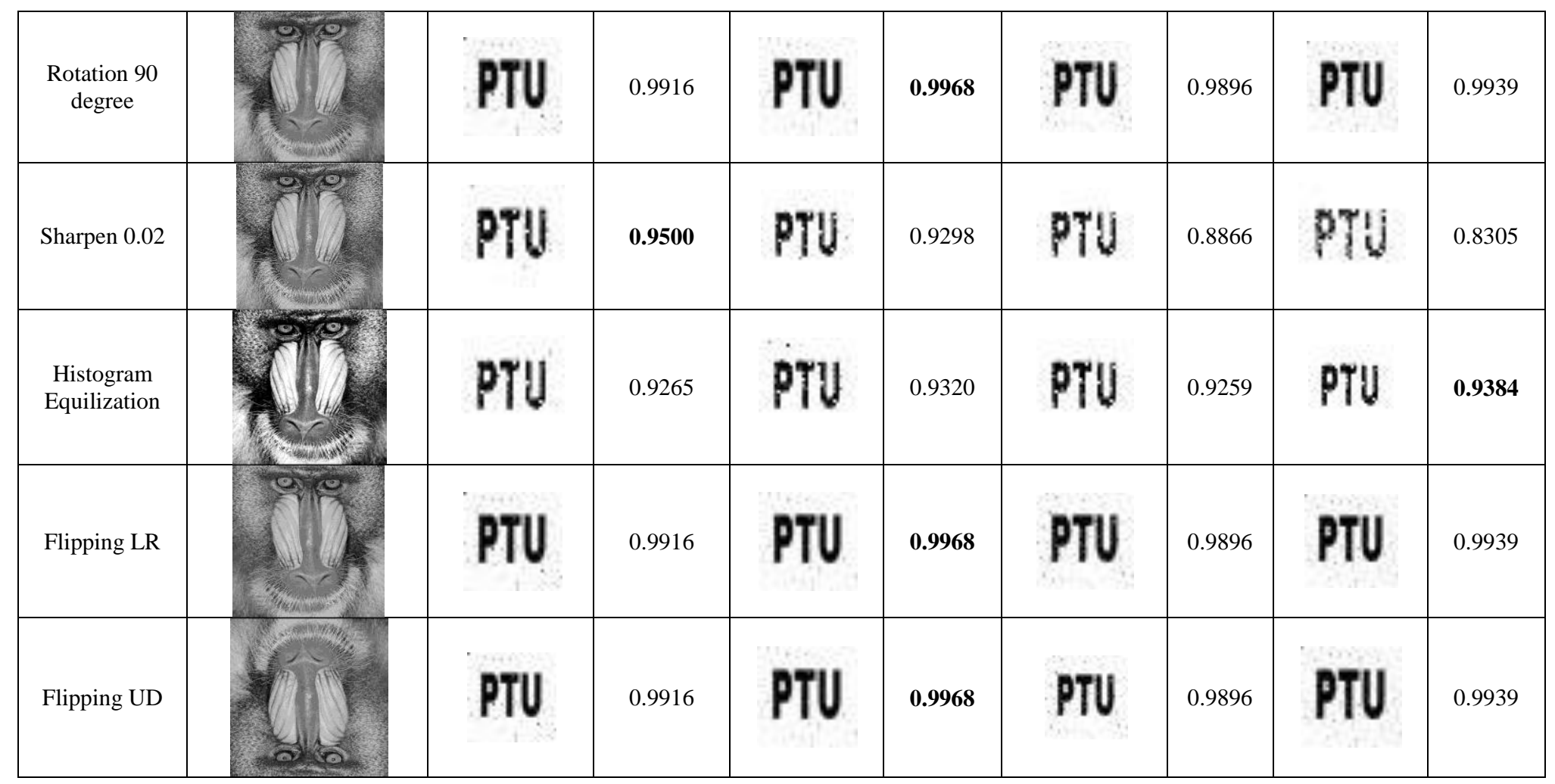

Table 2. Normalised Correlation of extracted watermarks from different locations after various attacks. (continued)

\begin{tabular}{|c|c|c|c|c|c|c|c|c|c|}
\hline \multirow{2}{*}{ Attack } & \multirow{2}{*}{$\begin{array}{c}\text { Attacked } \\
\text { Watermark Image }\end{array}$} & \multicolumn{2}{|c|}{ Attack Results at $(\mathbf{3 , 3})$} & \multicolumn{2}{|c|}{ Attack Results at $(\mathbf{4 , 4})$} & \multicolumn{2}{|c|}{ Attack Results at $(5,5)$} & \multicolumn{2}{|c|}{ Attack Results at $(6,6)$} \\
\hline & & $\begin{array}{l}\text { Extracted } \\
\text { watermark }\end{array}$ & $\mathrm{NC}$ & $\begin{array}{l}\text { Extracted } \\
\text { watermark }\end{array}$ & $\mathrm{NC}$ & $\begin{array}{l}\text { Extracted } \\
\text { watermark }\end{array}$ & $\mathrm{NC}$ & $\begin{array}{l}\text { Extracted } \\
\text { watermark }\end{array}$ & $\mathrm{NC}$ \\
\hline Crop & & & 0.7760 & & 0.8643 & & 0.8495 & & 0.9713 \\
\hline Random crop & & & 0.9369 & & 0.8635 & & 0.9268 & & 0.9462 \\
\hline Editing & & & 0.8791 & & 0.9169 & & 0.9545 & & 0.9084 \\
\hline $\begin{array}{c}\text { JPEG } \\
\text { compression }\end{array}$ & & & 0.9856 & & 0.9839 & & 0.9734 & & 0.9562 \\
\hline $\begin{array}{c}\text { JPEG } \\
\text { compression }\end{array}$ & & & 0.9829 & & 0.9878 & & 0.9595 & & 0.9468 \\
\hline
\end{tabular}




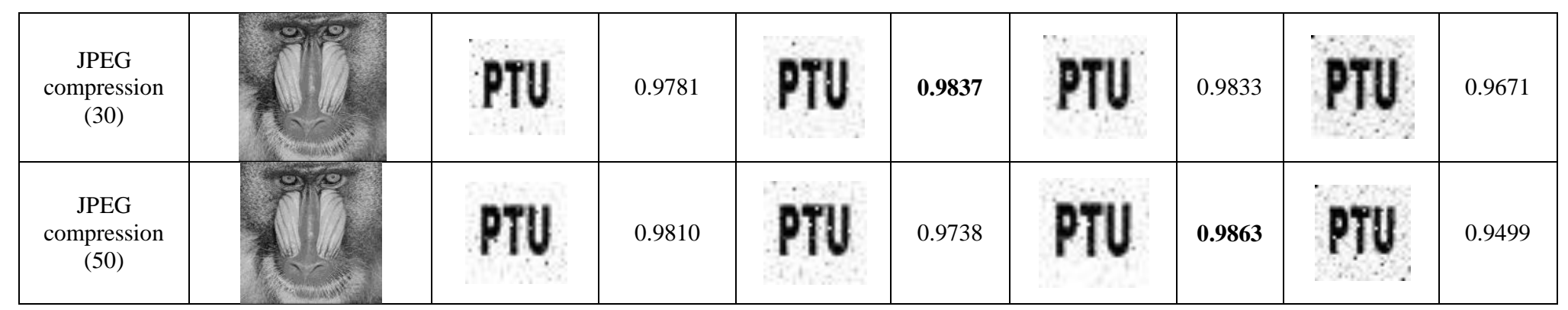

Table 2. Normalised Correlation of extracted watermarks from different locations after various attacks. (continued)

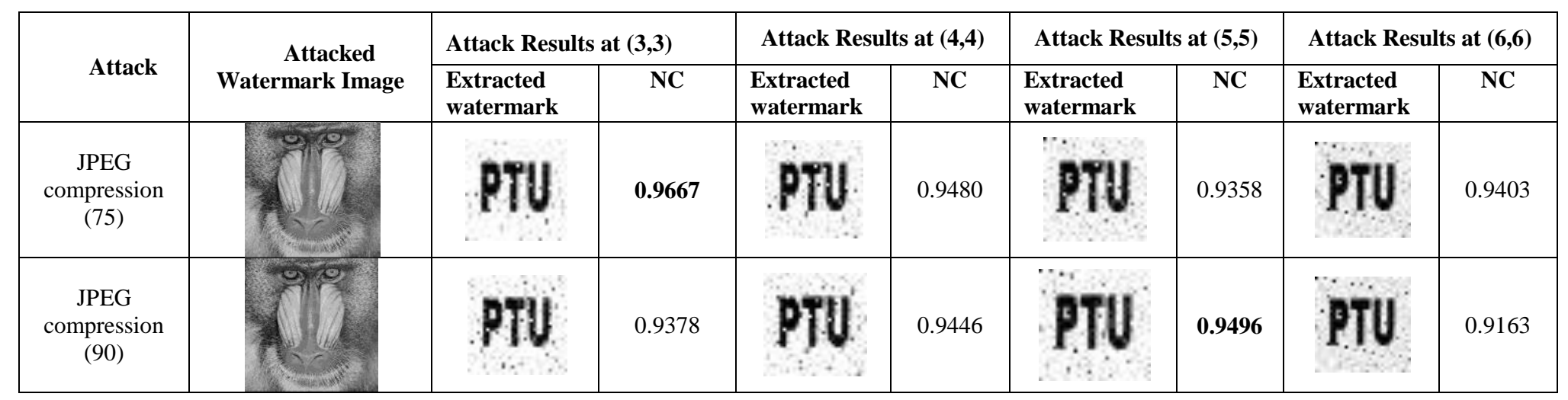

